\title{
N219Y, a new frequent mutation among mut forms of methylmalonic acidemia in Caucasian patients
}

\author{
Cecile Acquaviva ${ }^{1}$, Jean-François Benoist*, ${ }^{*}$, Isabelle Callebaut ${ }^{3}$, Nathalie Guffon ${ }^{5}$, \\ Hélène Ogier de Baulny ${ }^{4}$, Guy Touati ${ }^{6}$, Ahmet Aydin ${ }^{7}$, Dominique Porquet ${ }^{2}$ and \\ Jacques Elion ${ }^{1}$
}

\begin{abstract}
${ }^{1}$ Service de Biochimie-Génétique/INSERM U458, Assistance Publique-Hôpitaux de Paris, Hôpital Robert Debré, Paris, France; ${ }^{2}$ Service de Biochimie-Hormonologie, Assistance Publique-Hôpitaux de Paris, Hôpital Robert Debré, Paris, France; ${ }^{3}$ Systèmes moléculaires et biologie structurale, LMCP, CNRS UMR 7590, Universités Paris 6/7, France; ${ }^{4}$ Service de Neurologie et Maladies Métaboliques, Assistance Publique-Hôpitaux de Paris, Hôpital Robert Debré, Paris, France; ${ }^{5}$ Service de Pédiatrie, Hôpital Debrousse, 29 rue Soeur Bouvier, 69005 Lyon, France; ${ }^{6}$ Unité de maladies métaboliques, département de pédiatrie, Hôpital Necker Enfants-Malades, Paris, France; ${ }^{7}$ Department of paediatrics, Division of Metabolic Diseases and Nutrition, Cerrahpasa Medical Faculty, Istanbul, Turkey
\end{abstract}

Mutations in the MUT locus encoding for the methylmalonyl-CoA mutase (MCM) apoenzyme are responsible for the mut forms of methylmalonic acidemia (MMA). To date, 49 different mutations have been identified in mut MMA. Only two frequent mutations have been reported in the Japanese population and in AfricanAmericans. Here we report a new missense mutation N219Y (731 A $\rightarrow$ T) which we found in five unrelated families of French and Turkish descent. All the patients exhibited a severe mut phenotype and three of them were homozygotes for N219Y. Direct involvement of the mutation in the loss of enzyme activity was demonstrated by mutagenesis and transient expression study. Mapping of the mutation onto a threedimensional model of human MCM constructed by homology with the Propionibacterium shermanii enzyme shows that it lies in a highly conserved secondary structure motif and might suggest impaired folding and/or poor stability compatible with the mut phenotype. Finally, a 1\% N219Y carrier frequency was observed in a French anonymous control population. Thus, N219Y is the first frequent mut mutation to be reported in the Caucasian population. European Journal of Human Genetics (2001) 9, 577-582.

Keywords: methylmalonic acidemia; methylmalonyl-CoA mutase; mutation; Caucasian

\section{Introduction}

Methylmalonic acidemia (MMA; MIM\#251000) is an autosomal recessive inherited inborn error of metabolism that results from the functional impairment of methylmalonylCoA mutase (MCM, EC 5.4.99.2). MCM is a mitochondrial nuclear-encoded homodimer enzyme responsible for the conversion of L-methylmalonyl-CoA to succinyl-CoA in the

*Correspondence: J-F Benoist, Service de Biochimie-Hormonologie Hôpital Robert Debré, 48 Bd Sérurier, 75019 Paris, France.

Tel: +331400347 27; Fax: +331400347 90;

E-mail: jean-francois.benoist@rdb.ap-hop-paris.fr

Received 11 December 2000; revised 13 March 2001; accepted 2 May 2001
propionyl-CoA catabolic pathway toward the Krebs cycle. Each monomer binds one molecule of adenosylcobalamin, a vitamin $\mathrm{B}_{12}$ derivative and the cofactor of the enzymatic reaction.

MMA is a heterogeneous condition in which the $m u t$ forms (incidence estimated $1 / 30000$ to $1 / 50000$ ) are due to MCM apoenzyme deficiency. Studies on fibroblasts from patients with mut MMA have distinguished variant forms with undetectable residual MCM activity (designated $m u t^{\circ}$ ) and forms with hydroxocobalamin-dependent residual activity (designated $\left.m u t^{-}\right)^{1}$

In humans, MCM is encoded by a single gene (MUT) mapped on chromosome $6(6 \mathrm{p} 21)$. MUT consists of 13 exons spanning over $35 \mathrm{~kb}$ and producing a $2.7 \mathrm{~kb}$ mRNA. ${ }^{2}$ To date, 
molecular analysis of mut MMA has revealed at least 49 different mutations ${ }^{3-7}$ demonstrating the highly pleiomorphic nature of this condition. Only two mutations are frequent: the first one $(\mathrm{G} 717 \mathrm{~V})$ in African-Americans ${ }^{8}$ and the second (E117X) in the Japanese population. ${ }^{9,10}$

A three-dimensional model of the human MCM structure ${ }^{11}$ has been deduced from the crystal structure of the corresponding enzyme in Propionibacterium shermanii, that shares $65 \%$ sequence identity with human MCM. ${ }^{12}$ The model divides the molecule into two major domains: (i) a N-terminal eightstranded $\beta / \alpha$ barrel including the CoA moiety binding site and the dimer interface, and (ii) a C-terminal $\alpha / \beta$ domain with five parallel $\beta$ strands encompassing the ' $\mathrm{B}_{12}$-binding domain'. Availability of this model allows molecular modeling of the potential structural effects of the observed mutations.

Here, we report a novel missense mutation N219Y which, unexpectedly, was found in five independent $m u t^{\circ}$ Caucasian families. Site-directed mutagenesis and transient expression show that the observed nucleotide variation is indeed the causative mutation of the enzymatic defect. The affected asparagine residue is conserved among species and lies in a highly conserved secondary structure motif. Molecular modelling suggests that substitution by a tyrosine is likely to result in impaired folding and/or poor stability of the MCM molecule, compatible with the $m u t^{\circ}$ phenotype.

\section{Patients and methods \\ Patients}

We have investigated five unrelated patients affected with B12-unresponsive MMA. Patient 1 was the first child of consanguineous Turkish parents. He presented with acute metabolic acidosis at 3 months of age. However poor feeding and mild dehydration had been observed at 4 days of age. It was attributed to sepsis at this time but this was not documented and it could well have been the very first symptoms of the disease. His low protein diet consisted of $1 \mathrm{~g} / \mathrm{kg} / \mathrm{d}$ of protein, ie about $800 \mathrm{mg} / \mathrm{d}$ of valine. The patient died at 30 months of age following an acute decompensation. The four other patients were all born to French non consanguineous parents.

Patient 2 is affected with a neonatal onset form. She is presently 4 years old and has experienced six intercurrent decompensations. At 3 years of age, her valine tolerance was $700 \mathrm{mg} / \mathrm{d}$.

Patient 3 is a 10 year-old girl affected with a neonatal form. She has presented with two mild decompensations at 1 and 4 years of age, respectively. Valine tolerance at 3 years of age was $550 \mathrm{mg} / \mathrm{d}$.

Patient 4 presented with a neonatal decompensation. He is presently three and a half years of age and has experienced two mild and one severe intercurrent decompensations. His valine tolerance is $600 \mathrm{mg} / \mathrm{d}$.

Patient 5 is affected with a late onset form. She has experienced only one decompensation at 1 year of age. She is presently 5 years old. Her valine tolerance was $525 \mathrm{mg} / \mathrm{d}$ at 3 years of age.

\section{Biochemical phenotype}

Fibroblast cultures were obtained for each patient from skin biopsies. Cells were grown in HAM F10 medium with 12\% FCS under $5 \% \mathrm{CO}_{2}$. The mut MMA phenotype was established by measuring MCM activity as previously described. ${ }^{7}$ To discriminate $m u t^{\circ}$ from $m u t^{-}$phenotypes, the propionate incorporation test was performed. Fibroblasts were first cultured for 1 week in a cobalamin-free medium (MEM with $10 \%$ FCS under $5 \% \mathrm{CO}_{2}$ ). Then the incorporation of $\left[{ }^{14} \mathrm{C}\right]-$ propionate (NEN Life Science Products, Boston, MA, USA) into trichloroacetic acid-precipitable material was measured in cells grown for $18 \mathrm{~h}$ in the presence of increasing concentrations of hydroxocobalamin $(0,0.01,0.1$, and $1 \mu \mathrm{g} / \mathrm{mL}) .{ }^{13}$ Incorporation of $\left[{ }^{3} \mathrm{H}\right]$-phenylalanine (ICN Pharmaceuticals France, Orsay, France) was measured simultaneously as a control for cell number and rate of constitutive protein synthesis.

\section{Mutation detection}

MCM cDNA was prepared from cultured fibroblasts according to standard procedures. ${ }^{14}$ RT - PCR products obtained with the primers described by Ogasawara ${ }^{9}$ were directly sequenced on an ABI 310 automatic sequencer (Perkin Elmer, Foster City, CA, USA) using the Dye Terminator method, without the secondary nested-PCR included in the original procedure. To confirm the homozygous state of the N219Y mutation, the sequence of exon 3 was determined on genomic DNA using primers described by Adjalla. ${ }^{4}$ Moreover, to confirm the N219Y mutation by a second independent procedure and to screen a control population, a new PCR forward primer with a single base substitution was designed (primer 3mut, see legend to Figure 1) so as to create a NdeI restriction site when the mutation is present. Digestion of PCR-amplifed products from genomic DNA was performed according to the manufacturer's instructions (New England Biolabs, Beverly, MA, USA) and the resulting fragments analysed by electrophoresis on a $8 \%$ polyacrylamide gel.

\section{Transient expression study}

Firstly, a stable $m u t^{\circ}$ cell line (SCK2) was obtained by transforming MCM-deficient fibroblasts from patient 1 by the pLASwt plasmid (a gift from Prof A Fisher), that contains SV40 DNA encompassing the entire early region with a defective origin of replication. ${ }^{15}$ Fibroblasts were transfected by electroporation with a Gene Pulser (Biorad Laboratories, Hercules, CA, USA), using $4.10^{6}$ primary cultured cells, $4 \mu \mathrm{g}$ of pLASwt plasmid, and a $250 \mathrm{~V}$ pulse at a capacitance of $1600 \mu \mathrm{F}$. The SCK2 cell line was used for transient expression analysis.

Secondly, the $731 \mathrm{~A} \rightarrow \mathrm{T}$ substitution was introduced by sitedirected mutagenesis (USE Mutagenesis Kit-Amersham 


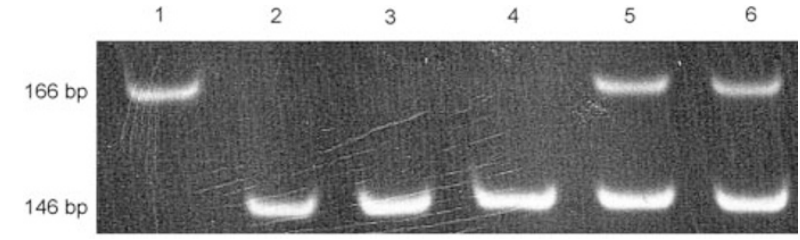

Figure 1 Detection of the $731 \mathrm{~A} \rightarrow \mathrm{T}$ substitution by $\mathrm{Ndel}$ restriction. Genomic DNA was PCR-amplified using the 3 mut forward primer (5' ARC TTA CTG GTA CCA TCC CA 3') localised just upstream from nucleotide 731 in exon 3. This primer contains a single nucleotide substitution (the underlined bold $C$ ) so as to create a Ndel restriction site when the $731 \mathrm{~A} \rightarrow T$ substitution is present. The reverse primer was that described by Adjalla et $a t^{4}$ for this exon. The products of Ndel digestion were analysed by electrophoresis on a $8 \%$ polyacrylamide gel. Wildtype sequence derived from a normal control (lane 1) gives a $166 \mathrm{bp}$ fragment. DNA from homozygous patients for the $731 \mathrm{~A} \rightarrow \mathrm{T}$ substitution are cleaved by Ndel giving rise to a $146 \mathrm{pb}$ fragment (lanes 2, 3, 4). In heterozygous patients, the two fragments are observed (lanes 5 and 6).

Pharmacia Biotech, Amersham, UK) into an expression vector, pCMVmut (constructed by $\mathrm{R}$ Touraine), containing the wild-type MCM cDNA downstream from the CMV promoter. Direct sequencing and enzymatic restriction were used to confirm the presence of the mutation in the recombinant plasmid (pCMVmut731A $\rightarrow \mathrm{T}$ ).

SCK2 cells were transfected using the Superfect reagent (Qiagen, Chatsworth, CA, USA) with: (i) the wild-type expression vector (pCMVmut) as a positive control; (ii) a vector containing a stop codon at position 238 (pCMVmutNSCD238) in the beginning of the coding sequence as a negative control; and (iii) pCMVmut731A $\rightarrow \mathrm{T}$. MCM activity was monitored $48 \mathrm{~h}$ after transfection, using the $\left[{ }^{14} \mathrm{C}\right]$-propionate incorporation method. All the experiments were carried out three times independently and in duplicate.

\section{Haplotypes}

Several microsatellite markers surrounding MUT on the short arm of the chromosome 6 were PCR-amplified and analysed on the ABI 310 sequencer. They included: D6S1714, D6S243, D6S465, D6S1669, and D6S1632.

\section{MCM structural modelisation}

To construct the three-dimensional structure of human MCM, molecular modelling simulations were performed with the MODELLER 4.0 software $^{16}$ using the experimental structure of $P$. shermanii MCM (Protein Data Bank accession code: 1REQ). As notified in Thomä and Leadlay, $^{11}$ the very high amino-acid sequence identity between human MCM and the $\alpha$ subunit of the $P$. shermanii enzyme allowed the construction of a 3D model that satisfies spatial constraints.

\section{Results}

All the patients' fibroblasts presented with a marked decrease in MCM enzyme activity, ie, less than $10 \%$ of the controls (Table 1). As activity is measured with an excess of cofactor (adenosylcobalamin concentration above $100 K_{m}$ ), this indicates that the defect indeed affects the apoenzyme and not the cofactor and that all the patients belong to the mut type. Similarly, propionate incorporation was drastically reduced in all cases and there was no correlation between added exogenous hydroxocobalamin and propionate uptake (Table 1). Altogether these data define a typical $m u t^{\circ} \mathrm{MMA}$ phenotype.

Comparison of DNA sequences obtained for the patients with the consensus sequence of the human MCM cDNA (Genebank, accession number M65022) revealed a novel single nucleotide substitution in MUT exon 3. It consists of an $\mathrm{A} \rightarrow \mathrm{T}$ transversion at position 731 in the cDNA. This change was confirmed by sequence determination and restriction analysis of PCR products from genomic DNA (Figure 1). Three patients were homozygotes for the substitution (patients 1 3 ) and two patients were heterozygotes (patients 4 and 5). As expected, for each homozygous patient, the parents are heterozygotes for the substitution (data not shown). Restriction analysis was also used to screen DNAs from two different control populations for the $731 \mathrm{~A} \rightarrow \mathrm{T}$ substitution: in a population of 105 anonymous mentally retarded children and in a control population of 100 anonymous normal children; it was found in the heterozygous state in two individuals from the first population.

For patients 4 and 5, a second sequence modification has been found. It consists in a $\mathrm{G} \rightarrow \mathrm{A}$ transition at position 399 and $a \mathrm{G} \rightarrow \mathrm{T}$ transition at position 549 in the cDNA, respectively. Interestingly, the $731 \mathrm{~A} \rightarrow \mathrm{T}$ substitution was found on chromosomes with various haplotypes. Indeed, except for the Turkish patient who was homozygote for all the studied markers, all the other patients including the two homozygotes for the $731 \mathrm{~A} \rightarrow \mathrm{T}$ substitution were heterozygotes for the microsatellite haplotype surrounding MUT.

At the protein level, the $731 \mathrm{~A} \rightarrow \mathrm{T}, 399 \mathrm{G} \rightarrow \mathrm{A}, 549 \mathrm{G} \rightarrow \mathrm{T}$ substitutions result respectively in the replacement of an asparagine residue by a tyrosine at position 219 (N219Y), an

Table 1 Patients' fibroblasts biochemical phenotypes

\begin{tabular}{lcccc}
\hline & \multicolumn{5}{c}{$\begin{array}{l}{\left[{ }^{14} \text { C]-propionate }\right.} \\
\text { incorporation }\end{array}$} \\
Patient & $\begin{array}{c}\text { MCM } \\
\text { enzymatic } \\
\text { activity }^{a}\end{array}$ & $\begin{array}{c}\text { Without } \\
\text { OHcbl }\end{array}$ & $\begin{array}{c}\text { OHcbl } \\
1 \mu \mathrm{g} / \mathrm{ml}\end{array}$ & $\begin{array}{c}\text { Biochemical } \\
\text { phenotype }^{\circ}\end{array}$ \\
\hline 1 & 30 & 4.5 & 4.5 & mut $^{\circ}$ \\
2 & $<10$ & 4.3 & 4.3 & mut $^{\circ}$ \\
3 & 10 & 0.9 & 0.9 & mut $^{\circ}$ \\
4 & 111 & 3.4 & 3.4 & mut $^{\circ}$ \\
5 & 46 & 2.1 & 2.0 & mut $^{\circ}$ \\
Controls & $1800 \pm 600, n=12$ & $80 \pm 20, n=10$ & \\
\hline
\end{tabular}

${ }^{\mathrm{a}} \mathrm{pmol} / \mathrm{mg}$ protein/min; ${ }^{\mathrm{b}} \mathrm{pmol}$ succinate/pmol phenylalanine 
arginine residue by a histidine at position 108 (R108H) and a glycine residue by a valine at position 158 (G158V). All this residues are conserved from bacteria to mouse and man, thus, although the R108H and G158V substitutions have not been studied functionally, it is likely that they represent the second deleterious mutation in patients 4 and 5 .
In the MCM primary structure, Asn219 is located in an amino-acid stretch that shows a particularly high level of homology between species (Figure 2). Mapping of the N219Y substitution on the three-dimensional model of human $\mathrm{MCM}^{11}$ shows that Asn219 is located in the fourth $\beta$-strand of the N-terminal eight-stranded $\beta / \alpha$ barrel, ie, a specific
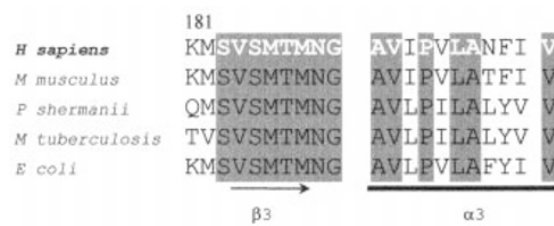
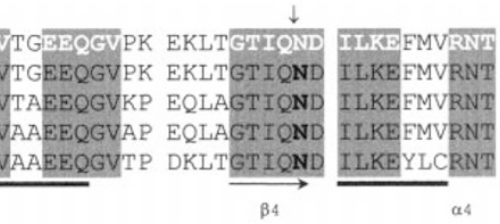

Figure 2 Partial alignment of methylmalonyl-CoA mutase amino acid sequence around asparagine 219 in various species. Sequences span amino acids 181 - 255 in Homo sapiens, Mus musculus, Propionibacterium shermanii, Mycobacterium tuberculosis and Escherichia coli enzymes (Swiss Prot accession numbers P22033, P16332, P11653, P71774 and P27253, respectively). Horizontal bars represent alpha helices and horizontal arrows beta-strands in the P. shermanii 3D structure. Position of asparagine 219 is indicated in bold and by a vertical arrow.

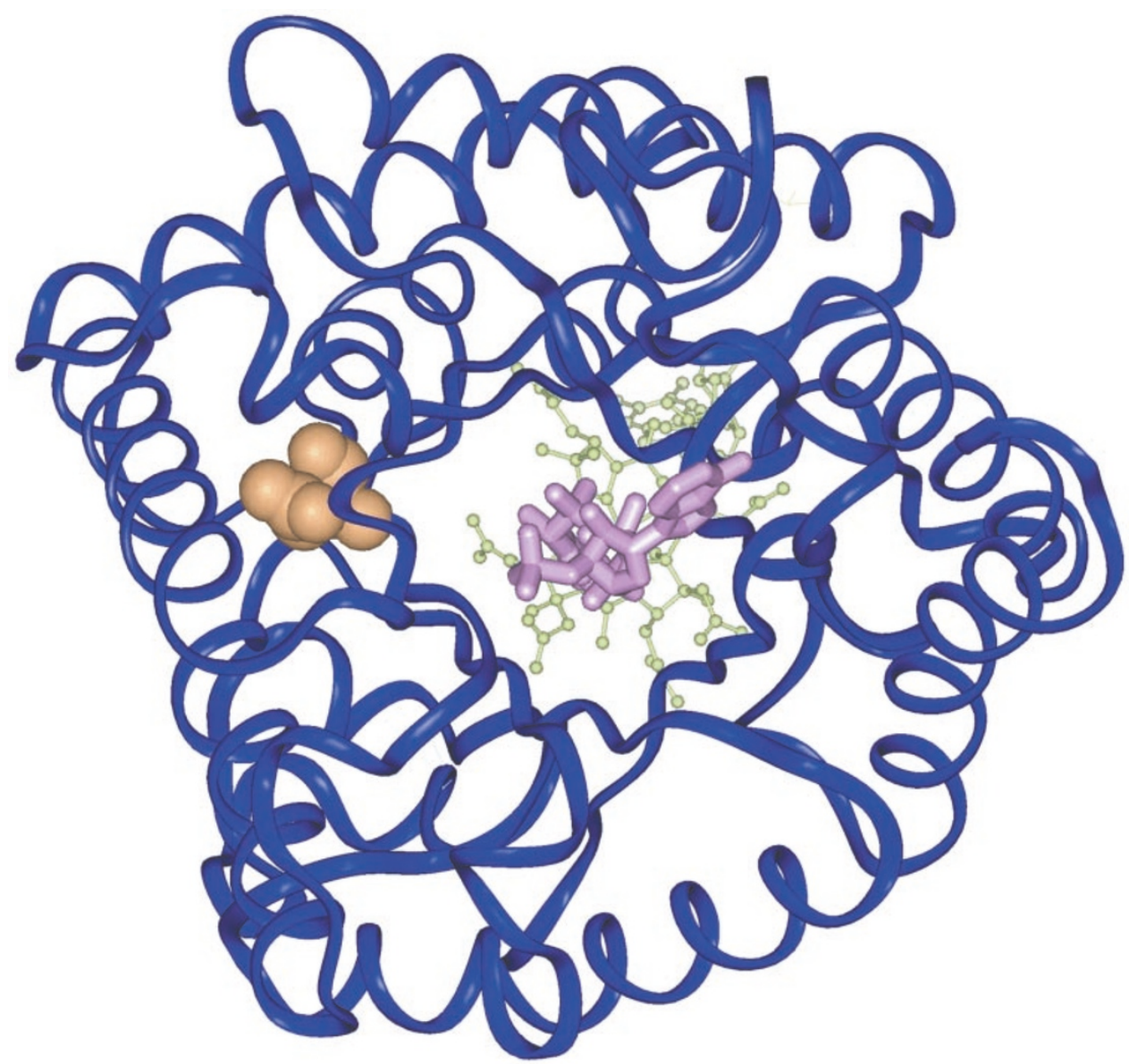

Figure 3 View of the three-dimensional structure of the $(\beta / \alpha)_{8}$ barrel of the human methylmalonyl-coenzyme A mutase model. The model was built on the basis of the experimental structure of the $\alpha$ chain of the Propionibacterium shermanii enzyme (PDB 1REQ). Domains are coloured as follows: blue for the $(\beta / \alpha)_{8}$ barrel which binds the substrate (desulfo-CoA, as existing in the 1REQ structure, in pink), and green for the cofactor adenosylcobalamin (in the back). Asparagine 195 (corresponding to Asn219 in human) is shown in a CPK representation and is buried between the $\beta$-barrel structure and one of the $\alpha$-helix surrounding this barrel. 
secondary structure motif of the molecule (Figure 3). However, it does not appear to have direct interaction with the substrate as its side chain is turned outside of the $\beta$-barrel and buried by one of the surrounding $\alpha$-helices.

To determine if the $731 \mathrm{~A} \rightarrow \mathrm{T}$ substitution was indeed responsible for the deficient phenotype, we performed transient expression studies in SCK2, a $m u t^{\circ}$ cell line immortalised from the fibroblasts of patient 1. As expected, transfection of the wild-type expression vector (pCMVmut) resulted in an increased propionate incorporation by SCK2 cells (Figure 4). In contrast, transfection of the vector

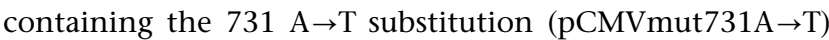
did not change propionate incorporation over the background value measured after transfection with pCMVmutNSCD238, a vector in which MCM cDNA contains a null mutation.

\section{Discussion}

Here, we report a novel mutation in the MUT gene which we observed in five independent Caucasian families with MMA. Insertion of this mutation in a wild-type MCM expression vector abolishes its ability to restore enzyme activity in a MCM-deficient cell line. Thus N219Y is indeed the causative mutation of the patients' $m u t^{\circ}$ phenotype. Residual MCM activity was very low in the patients' fibroblasts and all the patients were affected with a severe clinical form and a low valine tolerance $(<800 \mathrm{mg} / \mathrm{d})$. All experienced acute intercurrent decompensations and one was lethal in one patient. The $m u t^{\circ}$ phenotype observed in the heterozygous patients suggests that the second mutation involved is also of the mut type, although, to our knowledge, the phenotype resulting

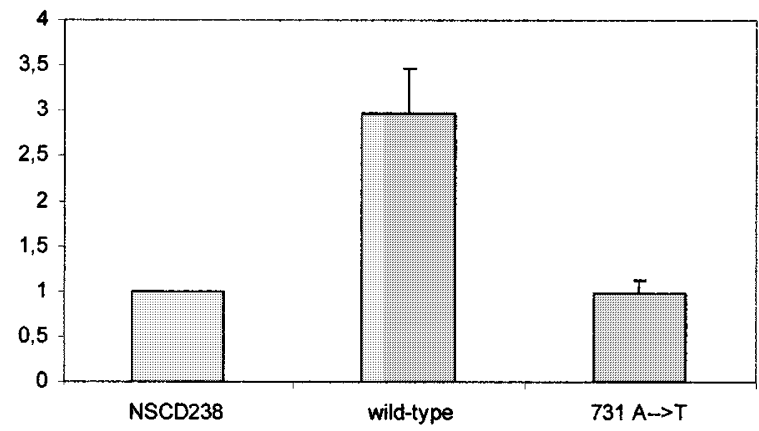

Figure 4 Transient expression of MCM activity in the SCK2 mut ${ }^{\circ}$ cell line. MCM activity was estimated by the $\left[{ }^{14} \mathrm{C}\right]$ propionate incorporation test during the transient phase of gene expression, $48 \mathrm{~h}$ after transfection of MCM cDNA containing vectors in SCK2 cells. pCMVmut is wild-type expression vector, pCMVmut731A $\rightarrow T$ the vector in which the $731 \mathrm{~A} \rightarrow T$ substitution has been introduced and pCMVmutNSCD238 a null expression vector. Results are expressed related to the basal activity obtained with pCMVmutNSCD238. The results shown are the mean and SD of three independent experiments performed in duplicate. from the $m u t^{\circ} / m u t^{-}$compound heterozygosity has not been precisely characterised.

Previous analysis of natural $m u t^{\circ}$ mutations on a homology model for human MCM has identified two types of mutations. Most of them affect a residue directly involved in catalysis, but modelling predicts that some mutations act rather by interfering with protein folding. ${ }^{11}$ Our modelling analysis of the N219Y mutation suggests that it belongs to this second type. Indeed, substitution of Asn219 by a Tyr residue might severely influence the overall MCM conformation in different ways. First, as this residue is located in a regular secondary structure motif (end of a beta-strand within the beta-barrel), it might result in an impaired folding process of this motif and/ or in a poor structure stability. Second and most importantly, Asn219 has PHI/PSI angle values typical of left-handed helix, a conformation almost exclusively occupied by glycine and asparagine residues. Substitution by a tyrosine should therefore inhibit the formation of such particular conformation. Finally, the much bulkier and hydrophobic Tyr side chain might hamper the positioning of the adjacent helix (due to a steric clash) and lead to the disruption of the whole $\mathrm{N}$ terminal beta/alpha barrel domain of the MCM molecule. The mut ${ }^{-}$mutations concern residues mainly located in the $\mathrm{B}_{12^{-}}$ binding domain. Few belong to the eight-stranded beta/alpha barrel domain but lie at the interface between these two domains that form a crevice which accommodates the adenosylcobalamin molecule. These mutations would alter the adenosylcobalamin binding. ${ }^{7}$

Genetic heterogeneity in mut MMA is high. Since the cloning of the MUT gene in 1988 by Ledley et al, ${ }^{17}$ at least 49 different mutations have been identified. All were reported in only one or two patients except for two (G717V and E117X) which are found in specific ethnic groups. G717V was found in four African-American and one Ghanaian MMA patients with the mut $^{-}$phenotype, suggesting that this mutation might be ancient and of African origin. ${ }^{8} \mathrm{E} 117 \mathrm{X}$ is a $m u t^{\circ}$ mutation and has been found with a high prevalence $(24 \%$ of the alleles tested) in a group of Japanese MMA patients. ${ }^{9,10}$ To our knowledge, no frequent mut mutation has been described to date in the Caucasian population. In our mut population (19 patients), the $\mathrm{N} 219 \mathrm{Y}$ mutation has been found in $21 \%$ of the alleles screened. The carrier frequency is even higher (57\%) among our seven $m u t^{\circ}$ patients. Four of the families in which we observed the N219Y mutation were of French descent. Screening an anonymous population of mentally retarded children living in France unexpectedly showed a 2\% frequency of N219Y carriers but none were found in a similarly sized-group of normal children. As there is no reason to expect that carriership for N219Y leads to mental retardation, we can consider a global carrier frequency around $1 \%$. This would suggest that N219Y is a frequent mutation in the French population and that the incidence of MMA in the French population is underestimated.

The finding of N219Y in a Turkish family indicates that $\mathrm{N} 219 \mathrm{Y}$ is also present in Caucasian populations from the 
Eastern part of the Mediterranean Basin. This could suggest an ancient origin to this mutation. Alternatively, the fact of finding the mutation on chromosome of different haplotypes may suggest independent origins to the mutation. Finer mapping of polymorphic markers around the mutation will be necessary to solve this issue.

\section{Acknowledgments}

We thank Professor A Fisher for providing the pLASwt vector and $M C$ Fondaneche for her technical assistance, Dr $R$ Touraine for providing the pCMVmut and the NSCD238 vectors. This work was supported by a grant from the Assistance Publique-Hôpitaux de Paris, CRC98014.

\section{References}

1 Fenton WA, Gravel RA, Rosenblatt DS: Disorders of propionate and methylmalonate metabolism. In: Scriver CR, Beaudet AL, Valle D, Sly WS (eds): The metabolic and molecular bases of inherited disease. 8th Ed. New York: McGraw-Hill, 2001, pp. $2165-2193$

2 Nham SU, Wilkemeyer MF, Ledley FD: Structure of the human methylmalonyl-CoA mutase (MUT) locus. Genomics 1990; 8: $710-716$.

3 Ledley FD, Rosenblatt DS: Mutations in mut methylmalonic acidemia: clinical and enzymatic correlations. Hum Mutat 1997; 9: $1-6$.

4 Adjalla CE, Hosack AR, Gilfix BM et al: Seven novel mutations in mut methylmalonic aciduria. Hum Mutat 1998; 11: 270-274.

5 Heptinstall LE, Garside HJ, Till J, Walter JH, Wraith JE, Besley GTN: Mutation analysis in mutase-deficient methylmalonic aciduria. [Abstract P102] J Inher Metab Dis 1999; S1: 94.

6 Fuchshuber A, Mucha B, Baumgartner ER, Vollmer M, Hildebrandt $\mathrm{F}$ : $m u t^{\circ}$ methylmalonic acidemia: eleven novel mutations of the methylmalonyl-CoA mutase including a deletioninsertion mutation. Hum Mutat 2000; 16: 179.

7 Benoist JF, Acquaviva C, Callebaut I et al: Molecular and structural analysis of two novel mutations in a patient with mut- methylmalonyl-CoA deficiency. Mol Genet Metab 2001; 72: $181-184$.
8 Adjalla CE, Hosack AR, Matiaszuk NV, Rosenblatt DS: A common mutation among blacks with mut- methylmalonic aciduria. Hum Mutat 1998; S1: S248-S250.

9 Ogasawara M, Matsubara Y, Mikami H, Narisawa K: Identification of two novel mutations in the methylmalonyl-CoA mutase gene with decreased levels of mutant mRNA in methylmalonic acidemia. Hum Mol Genet 1994; 3: 867-872.

10 Mikami H, Ogasawara M, Matsubara Y et al: Molecular analysis of methylmalonyl-CoA mutase deficiency: identification of three missence mutations in mut0 patients. J Hum Genet 1999; 44: $35-39$

11 Thomä NH, Leadlay PF: Homology modeling of human methylmalonyl-CoA mutase: a structural basis for point mutations causing methylmalonic aciduria. Protein Science 1996; 5: $1922-1927$.

12 Mancia F, Keep NH, Nakagawa A et al: How coenzyme B12 radicals are generated: the crystal structure of methylmalonylcoenzyme A mutase at $2 \AA$ resolution. Structure 1996; 4: 339 _ 350.

13 Willard HF, Ambani LM, Hart AC, Mahoney MJ, Rosenberg LE: Rapid prenatal and postnatal detection of inborn errors of propionate methylmalonate and cobalamin metabolism: a sensitive assay using cultured cells. Hum Genet 1976; 34: 277 283.

14 Sambrook J, Fritsch EF, Maniatis T: Molecular cloning: a laboratory manual 2nd edition. Cold Spring Harbor 1989.

15 Daya-Grosjean L, James MR, Drougard C, Sarasin A: An immotalized xeroderma pigmentosum, group C, cell line which replicates SV 40 shuttle vectors. Mutation Res 1987, 183: $185-$ 196.

16 Sali A, Blundell TL: Comparative protein modeling by satisfaction of spatial restraints. J Mol Biol 1993; 234: 779-815.

17 Ledley FD, Lumetta MR, Zoghbi HY, Van Tuinen P, Ledbetter SA, Ledbetter DH: Mapping of human methylmalonyl-CoA mutase (MUT) locus on chromosome 6. Am J Hum Genet 1988; 42: 839 846 